Irish Section Meeting, 21-23 June 2017, What governs what we eat?

\title{
Consumer exposure to nutrition and health claims in Ireland
}

\author{
D. McGettigan, M.A. Kerr and M.T. McCann \\ Northern Ireland Centre for Food and Health (NICHE), Ulster University, Coleraine, BT52 1SA, UK
}

Nutrition and health claims (NHC) are used on food products by manufacturers to highlight the proven health benefits of a product ${ }^{(1)}$. Evidence suggests that the presence of a NHC on a food product may help increase sales ${ }^{(2)}$ making heir use attractive to food manufacturers. The prevalence of NHC made on foods is rising ${ }^{(3)}$ as industry has recognised their value as a marketing tool ${ }^{(4)}$. This study aimed to examine consumer exposure to NHC on pre-packaged foods and beverages in Ireland.

An observational analysis was undertaken to assess the labels of pre-packaged foods and beverages available in stores in Ireland to determine the prevalence and type of NHC. Where present, a photograph of the packaging was taken and the details of the claim recorded. Foods were categorised into 13 food groups. NHC were categorised in accordance with EU Regulation 1924/2006 on nutrition and health claims made on foods.

Three retail outlets which were representative of nationwide stores across the Island of Ireland were assessed; one major retailer, one medium independent retailer and one discount retailer. Three hundred and ninety three food products were randomly sampled from these retail outlets stocklists.

Of the 393 products sampled, $36.4 \%$ carried at least one nutrition or health claim. $29.3 \%$ of products carried at least one nutrition claim and $8.4 \%$ at least one health claim. The discount retailer was found to have the highest proportion of nutrition and health claims (41.1\% of products) with the major retailer and medium independent having $37.7 \%$ and $29.3 \%$ respectively. Of all the claims observed, $63 \%$ were nutrition claims and $20.1 \%$ were health claims.

In Ireland while NHC prevalence appears to have decreased since $2010^{(5)}$, consumers remain exposed to a higher number of NHC in comparison to other EU countries ${ }^{(6)}$. The high level of consumer exposure to NHC in Ireland may be perceived as beneficial due to their role in facilitating healthy food choices.

1. Hughes C, Wellard L, Lin J et al. (2013) Public Health Nutr 16(12), 2154-2162.

2. National Starch and Chemical GmbH (2007) Nutraingredients 24 July.

3. EFSA (2015a) Nutrition and health claims Available from: http://www.efsa.europa.eu/en/topics/topic/nutrition

4. Van Buul VJ and Brouns FJ (2015) Crit Rev Food Sci Nutr 55(11), 1552-1560.

5. Lalor F, Kennedy J, Flynn MA et al. (2010) Public Health Nutr 13(05), 704-711.

6. Hieke S, Kuljanic N, Pravst I et al. (2016) Nutrients 8(3), 137. 